\title{
Mathematical Study of Impact of Travel Rates of Humans in the Transmission of Dengue Disease
}

\author{
Ganga Ram Phaijoo ${ }^{1 *}$ and Dil B. Gurung ${ }^{2}$ \\ ${ }^{1}$ Department of Mathematics, School of Science \\ ${ }^{2}$ Kathmandu University, Dhulikhel, Kavre, Nepal \\ *Corresponding Author \\ gangaram@ku.edu.np
}

\begin{abstract}
Dengue is an infectious disease caused by dengue viruses of four serotypes DEN 1 to DEN 4. It is transmitted to humans by the bite of female Aedes mosquitoes. The disease has become a significant public health concern in recent years. Dengue viruses have expanded their geographic range through the movement of infected humans. In the present work, a two-patch epidemic model is proposed to describe the transmission dynamics of dengue disease in patches with the mobility of humans. Different travel rates of humans are considered to study the dynamics of the disease. Different patches are considered to have different disease prevalence. Basic reproduction number of the model is calculated, and some threshold conditions are established to study local stability of the equilibrium points. The travel of humans can help the dengue disease to increase its prevalence and sometimes the travel can be helpful in controlling the spread of the disease. Present work demonstrates that proper control in travel rates is helpful in reducing the spread and dominancy of the disease.
\end{abstract}

\section{Keywords}

Dengue disease, patch, basic reproduction number, equilibrium point, local stability

\section{Introduction}

Dengue is the most common mosquito-borne infectious disease caused by four different serotypes of viruses DEN 1 to DEN 4. The disease is transmitted to humans by the bites of infected female Aedes mosquitoes. An estimated 2.5 billion people live in the areas where the dengue is endemic. Dengue has been recognized in over 100 countries, and an estimated 50 - 100 million dengue cases occur annually (World Health Organization 2012). A person infected of one of the serotypes of the dengue viruses gets recovered having lifelong immunity to that serotype and becomes susceptible to get infected by other serotypes of viruses. About 12 weeks, the person becomes more susceptible to develop dengue hemorrhagic or dengue shock syndrome (Gubler 1998).

Kermack and Mckendrick (1927) developed a SIR (Susceptible, Infectious, Removals) model in a closed population (no immigration or emigration), which became very famous in studying the dynamics of infectious diseases. Mathematical models help to obtain a better understanding of the transmission mechanism of the disease. The models help to identify the features that are most influent in the transmission of the disease. So, many mathematical models have been used to examine the transmission dynamics of dengue fever, (Esteva and Vargas 1998; Esteva and Vargas 1999; Soewono and Supriatna 2001; Maidan and Yang 2007; Pongsumpun 2008; Pinho, et al., 2010; Kongnuy, et al., 2011; Gakkhar and Chavda 2013; Phaijoo and Gurung 2015).

Travelling of the people from one place to another place contribute to spreading the disease in new places. Many mathematical models with the spatial spread of the diseases (Arino and Driessche 2003; Murray 2003; Wang and Mulone 2003; Wang and Zhao 2004; Arino and Driessche 2006) are proposed to study the transmission dynamics of infectious diseases. In the present work, we have formulated a SIR - SI deterministic two- 
patch model. Ying Hen et al. (2007) had used such a patch model to study the diseases which are transmitted to humans from humans directly.

The present model is developed to study the transmission dynamics of dengue disease, which is not transmitted to humans from humans directly; it is transmitted to humans by female Aedes mosquitoes.

\section{Model Formulation}

We consider SIR (Susceptible, Infectious, and Recovered) compartments for humans and SI (Susceptible, Infectious) compartments for mosquitoes. The recovered compartment of mosquito's population is not considered due to their short life period.

Let $S_{1}^{h}, I_{1}^{h}, R_{1}^{h}, S_{1}^{v}, I_{1}^{v}, N_{1}^{h}, N_{1}^{v}$ and $S_{2}^{h}$, $I_{2}^{h}, R_{2}^{h}, S_{2}^{v}, I_{2,}^{v}, N_{2}^{h}, N_{2}^{v}$ respectively denote the number of susceptible humans, infective humans, recovered humans, susceptible mosquitoes, infective mosquitoes, total human population and total mosquito population in patch 1 and patch 2 respectively. Human (host) population is assumed to travel between the patches. Mosquito (vector) population is assumed not to travel between patches due to their short-distance flight capacity.

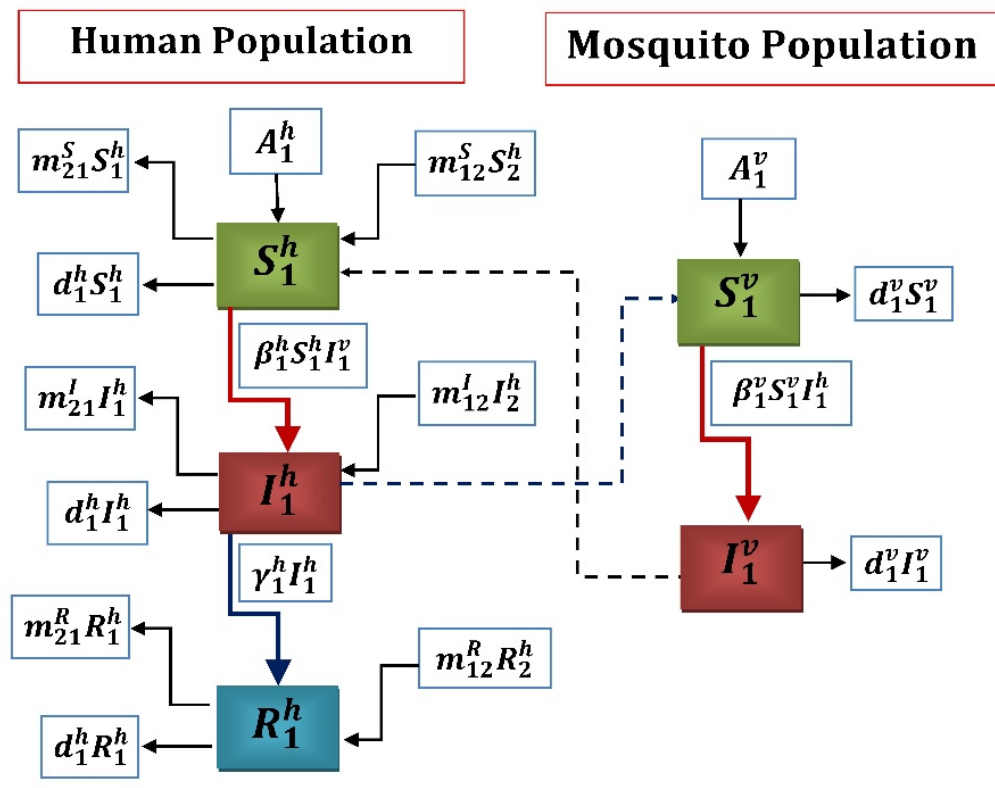

Fig. 1. Flow Chart for patch 1 of the two-patch model of dengue disease.

The two-patch model which describes the dengue disease transmission between two patches with the mobility of humans is given by 


$$
\begin{aligned}
& \frac{d S_{1}^{h}}{d t}=A_{1}^{h}-\beta_{1}^{h} S_{1}^{h} I_{1}^{v}-d_{1}^{h} S_{1}^{h}+m_{12}^{S} S_{2}^{h}-m_{21}^{S} S_{1}^{h} \\
& \frac{d I_{1}^{h}}{d t}=\beta_{1}^{h} S_{1}^{h} I_{1}^{v}-\left(\gamma_{1}^{h}+d_{1}^{h}\right) I_{1}^{h}+m_{12}^{I} I_{2}^{h}-m_{21}^{I} I_{1}^{h} \\
& \frac{d R_{1}^{h}}{d t}=\gamma_{1}^{h} I_{1}^{h}-d_{1}^{h} R_{1}^{h}+m_{12}^{R} R_{2}^{h}-m_{21}^{R} R_{1}^{h} \\
& \frac{d S_{1}^{v}}{d t}=A_{1}^{v}-\beta_{1}^{v} S_{1}^{v} I_{1}^{h}-d_{1}^{v} S_{1}^{v} \\
& \frac{d I_{1}^{v}}{d t}=\beta_{1}^{v} S_{1}^{v} I_{1}^{h}-d_{1}^{v} I_{1}^{v} \\
& \frac{d S_{2}^{h}}{d t}=A_{2}^{h}-\beta_{2}^{h} S_{2}^{h} I_{2}^{v}-d_{2}^{h} S_{2}^{h}+m_{21}^{S} S_{1}^{h}-m_{12}^{S} S_{2}^{h} \\
& \frac{d I_{2}^{h}}{d t}=\beta_{2}^{h} S_{2}^{h} I_{2}^{v}-\left(\gamma_{2}^{h}+d_{2}^{h}\right) I_{2}^{h}+m_{21}^{I} I_{1}^{h}-m_{12}^{I} I_{2}^{h} \\
& \frac{d R_{2}^{h}}{d t}=\gamma_{2}^{h} I_{2}^{h}-d_{2}^{h} R_{2}^{h}+m_{21}^{R} R_{1}^{h}-m_{12}^{R} R_{2}^{h} \\
& \frac{d S_{2}^{v}}{d t}=A_{2}^{v}-\beta_{2}^{v} S_{2}^{v} I_{2}^{h}-d_{2}^{v} S_{2}^{v} \\
& \frac{d I_{2}^{v}}{d t}=\beta_{2}^{v} S_{2}^{v} I_{2}^{h}-d_{2}^{v} I_{2}^{v}
\end{aligned}
$$

where, $S_{1}^{h}+I_{1}^{h}+R_{1}^{h}=N_{1}^{h}, S_{2}^{h}+I_{2}^{h}+R_{2}^{h}=N_{2}^{h}, S_{1}^{v}+I_{1}^{v}=N_{1}^{v}, S_{2}^{v}+I_{2}^{v}=N_{2}^{v}$

And,

$$
\beta_{1}^{h}=\frac{b \eta_{1}^{h}}{N_{1}^{h}}, \quad \beta_{2}^{h}=\frac{b \eta_{2}^{h}}{N_{2}^{h}}, \beta_{1}^{v}=\frac{b \eta_{1}^{v}}{N_{1}^{h}}, \quad \beta_{2}^{v}=\frac{b \eta_{2}^{v}}{N_{2}^{h}}
$$

The total host and vector population sizes in two patches are

$$
N^{h}=N_{1}^{h}+N_{2}^{h}, \quad N^{v}=N_{1}^{v}+N_{2}^{v}
$$

\section{Parameters used in the model}

\section{Parameters}

\section{Descriptions}

$\boldsymbol{d}_{1}^{h}, \boldsymbol{d}_{2}^{h} \quad$ death rates of host population in patch 1 and patch 2

$\boldsymbol{d}_{\mathbf{1}}^{v}, \boldsymbol{d}_{2}^{v} \quad$ death rates of vector population in patch 1 and patch 2

$\gamma_{1}^{h}, \gamma_{2}^{h} \quad$ recovery rates of host population in patch 1 and patch 2

$\boldsymbol{\eta}_{\mathbf{1}}^{\boldsymbol{h}}, \boldsymbol{\eta}_{\mathbf{2}}^{\boldsymbol{h}} \quad$ transmission probabilities from vector to host in patch 1 and patch 2

$\boldsymbol{\eta}_{\mathbf{1}}^{v}, \boldsymbol{\eta}_{2}^{v} \quad$ transmission probabilities from host to vector in patch 1 and patch 2

$\boldsymbol{b}, \boldsymbol{b} \quad$ biting rates of vectors in patch 1 and patch 2

$A_{1}^{h}, A_{2}^{h} \quad$ recruitment rates of host population in patch 1 and patch 2

$\boldsymbol{A}_{\mathbf{1}}^{v}, \boldsymbol{A}_{\mathbf{2}}^{v} \quad$ recruitment rates of vector population in patch 1 and patch 2

$\boldsymbol{m}_{21}^{S, I, R}, \boldsymbol{m}_{12}^{S, I, R} \quad$ Travel rates of susceptible $(S)$, infective $(I)$ and recovered $(R)$ host from patch 1 to patch 2 , and patch 2 to patch 1 
Theorem 1: Disease-free equilibrium of the model (2.1) exists and is unique.

Proof: A disease free equilibrium (DFE) for the system (2.1) is a steady state solution of the system where $I_{1}^{h}=0, I_{2}^{h}=0, I_{1}^{v}=0$ and $I_{2}^{v}=0$.

In disease free situation,

$$
\begin{aligned}
& \frac{d R_{1}^{h}}{d t}=-d_{1}^{h} R_{1}^{h}+m_{12}^{R} R_{2}^{h}-m_{21}^{R} R_{1}^{h} \\
& \frac{d R_{2}^{h}}{d t}=-d_{2}^{h} R_{2}^{h}+m_{21}^{R} R_{1}^{h}-m_{12}^{R} R_{1}^{h}
\end{aligned}
$$

In matrix form, above system (2.2) can be written as

$$
-\zeta R^{h}=0
$$

where,

$$
\zeta=\left[\begin{array}{cc}
d_{1}^{h}+m_{21}^{R} & -m_{12}^{R} \\
-m_{21}^{R} & d \_2^{\wedge} h+m_{12}^{R}
\end{array}\right], R^{h}=\left[R_{1}^{h}, R_{2}^{h}\right]^{T}
$$

Here, $\zeta$ has all off-diagonal entries negative, and both columns have a positive-sum. So, $\zeta$ must be an irreducible and non-singular $M$ - matrix (Berman and Plemmons (1979)) and the system (2.3) has a unique solution. $R^{h}=0$ is the solution to the system. i.e., $R_{1}^{h}=0, R_{2}^{h}=0$.

Hence, in disease free situation, $I_{1}^{h}=0, I_{2}^{h}=0, I_{1}^{v}=0, I_{2}^{v}=0, R_{1}^{h}=0, R_{2}^{h}=0$ and so, $S_{1}^{h}=$ $N_{1}^{h}, S_{2}^{h}=N_{2}^{h}, S_{1}^{v}=N_{1}^{v}, S_{2}^{v}=N_{2}^{v}$.

In disease-free situation, from the system of equations (2.1);

$$
\left[\begin{array}{cc}
d_{1}^{h}+m_{21}^{S} & -m_{12}^{S} \\
-m_{21}^{S} & d_{2}^{h}+m_{12}^{S}
\end{array}\right]\left[\begin{array}{c}
N_{1}^{h} \\
N_{2}^{h}
\end{array}\right]=\left[\begin{array}{c}
A_{1}^{h} \\
A_{2}^{h}
\end{array}\right]
$$

i.e.,

(For the host populations only)

where,

$$
C S^{h}=A^{h}
$$

And,

$$
C=\left[\begin{array}{cc}
d_{1}^{h}+m_{21}^{S} & -m_{12}^{S} \\
-m_{21}^{S} & d_{2}^{h}+m_{12}^{S}
\end{array}\right], S^{h}=\left[\begin{array}{c}
N_{1}^{h} \\
N_{2}^{h}
\end{array}\right], A^{h}=\left[\begin{array}{c}
A_{1}^{h} \\
A_{2}^{h}
\end{array}\right]
$$

$$
\left[\begin{array}{cc}
d_{1}^{v} & 0 \\
0 & d_{2}^{v}
\end{array}\right]\left[\begin{array}{c}
N_{1}^{v} \\
N_{2}^{v}
\end{array}\right]=\left[\begin{array}{l}
A_{1}^{v} \\
A_{2}^{v}
\end{array}\right]
$$

i.e.,

(For the vector populations only)

where,

$$
D S^{v}=A^{v}
$$

Matrix $C$ has positive column sums, and each non-diagonal element is negative. So, matrix $C$ is an irreducible and non-singular $M$ - matrix. Hence, $C^{-1}$ exists and $C^{-1}>0$ (Berman and Plemmons (1979)). Thus, there is a unique solution, $S^{h}=C^{-1} A^{h}>0$. 
$D$ is a diagonal matrix with positive diagonal elements $d_{1}^{v}, d_{2}^{v}$. So, $D^{-1}$ exists and is non-negative. So, $S^{v}=D^{-1} A^{v}$ is a unique solution to $D S^{v}=A^{v}$. The results show that there always exists a unique disease-free equilibrium point.

\section{Basic reproduction number}

The basic reproduction number $R_{0}$ is defined as the average number of secondary infections that occur when one infective is introduced into an utterly susceptible host population.

For the computation of the basic reproduction number we order the infected variables by $I_{1}^{h}, I_{2}^{h}, I_{1}^{v}$, $I_{2}^{v}$ and use the Next Generation Matrix method [20, 21] (Diekmann et al. (1990), Driessche and Watmough (2002)). Then,

where,

$$
F=\left[\begin{array}{cc}
0 & F_{12} \\
F_{21} & 0
\end{array}\right], \quad V=\left[\begin{array}{cc}
V_{11} & 0 \\
0 & V_{22}
\end{array}\right]
$$

$$
\begin{aligned}
& F_{12}=\left[\begin{array}{cc}
\beta_{1}^{h} N_{1}^{h} & 0 \\
0 & \beta_{2}^{h} N_{2}^{h}
\end{array}\right], \quad F_{21}=\left[\begin{array}{cc}
\beta_{1}^{v} N_{1}^{v} & 0 \\
0 & \beta_{2}^{v} N_{2}^{v}
\end{array}\right] \\
& V_{11}=\left[\begin{array}{cc}
m_{21}^{I}+\gamma_{1}^{h}+d_{1}^{h} & -m_{12}^{I} \\
-m_{21}^{I} & m_{12}^{I}+\gamma_{2}^{h}+d_{2}^{h}
\end{array}\right], \quad V_{22}=\left[\begin{array}{cc}
d_{1}^{v} & 0 \\
0 & d_{2}^{v}
\end{array}\right]
\end{aligned}
$$

Matrix $V_{11}$ has positive column sums, and all off-diagonal elements are negative. So, the matrix $V_{11}$ is an irreducible non-singular $M$ - matrix. Hence, $V_{11}^{-1}$ exists and is positive, i.e., $V_{11}^{-1}>0$.

$V_{22}$ is a diagonal matrix with positive entries. So, $V_{22}^{-1}$ exists and is non-negative. The basic reproduction number, $R_{0}$, for the system (2.3) is the spectral radius of $F V^{-1}$.

where,

$$
\therefore R_{0}=\frac{1}{2} \sqrt{2 a\left(R_{01}^{2}+R_{02}^{2}\right)+2\left[a^{2}\left(R_{02}^{2}-R_{01}^{2}\right)^{2}+4 a R_{01}^{2} R_{02}^{2}\right]^{1 / 2}}
$$

$$
\begin{gathered}
R_{01}=\sqrt{\frac{\beta_{1}^{h} \beta_{1}^{v} N_{1}^{h} N_{1}^{v}}{d_{1}^{v}\left(\gamma_{1}^{h}+d_{1}^{h}+m_{21}^{I}\right)}}(\text { Basicreproduction number of patch 1) } \\
R_{02}=\sqrt{\frac{\beta_{2}^{h} \beta_{2}^{v} N_{2}^{h} N_{2}^{v}}{d_{2}^{v}\left(\gamma_{2}^{h}+d_{2}^{h}+m_{12}^{I}\right)}}(\text { Basic reproduction number of patch } 2) \\
a=1+\frac{m_{12}^{I} m_{21}^{I}}{d_{1}^{h} d_{2}^{h}+d_{1}^{h} \gamma_{2}^{h}+d_{1}^{h} m_{12}^{I}+\gamma_{1}^{h} d_{2}^{h}+\gamma_{1}^{h} \gamma_{2}^{h}+\gamma_{1}^{h} m_{12}^{I}+m_{21}^{I} d_{2}^{h}+m_{21}^{I} \gamma_{2}^{h}}
\end{gathered}
$$

Theorem 2: If $R_{0}<1$, then the disease-free equilibrium is locally asymptotically stable and unstable if $R_{0}>1$.

Proof: Let $J_{11}$ and $J_{12}$ be the matrices of partial derivatives evaluated at the diseasefree equilibrium. The Jacobian matrix for the linearization of the system about the diseasefree equilibrium is obtained as the block structure

$$
J=\left[\begin{array}{cc}
J_{11} & J_{12} \\
0 & F-V
\end{array}\right]
$$

The matrix is upper triangular. So, the eigenvalues of $J$ are those of the partition matrices $J_{11}$ and $F-V$. Also,

$$
J_{11}=\left[\begin{array}{cc}
-C & 0 \\
0 & -D
\end{array}\right]
$$

The matrices $C$ and $D$ (matrices defined in theorem 1) are non-singular $M$-matrices. So, spectral abscissa $s(-C)<0, s(-D)<$ 0 (Berman and Plemmons (1979)) and eigenvalues of the matrix $J_{11}$ have negative real parts.

Hence, the matrix $J$ will have eigenvalues all with negative real parts if the matrix $F-V$ has all eigenvalues with negative real parts. Also, $F$ is a non-negative matrix and $V$ is non-singular $M$-matrix. So, eigenvalues of $F-V$ will have negative real parts if and only if $\rho\left\{F V^{-1}\right\}<1$ (Driessche and Watmough (2002)). i.e., the disease-free equilibrium is locally asymptotically stable if and only if the 
basic reproduction number, $R_{0}=\rho\left\{F V^{-1}\right\}<$ 1.

If $R_{0}>1$, then $s(F-V)>0$. It shows that at least one eigenvalue lies in the right halfplane. So, the disease equilibrium is unstable if $R_{0}>1$.

\section{For Patch 1}

Theorem 3: The disease-free equilibrium point is locally asymptotically stable if $R_{0}<$ 1 and unstable if $R_{01}>1$.

Proof: As both host and vector populations are considered constant, we can consider only the following 3 equations for patch 1

$$
\begin{gathered}
\frac{d S_{1}^{h}}{d t}=A_{1}^{h}-\beta_{1}^{h} S_{1}^{h} I_{1}^{v}-d_{1}^{h} S_{i}^{h}+m_{12}^{S} S_{2}^{h}-m_{21}^{S} S_{1}^{h} \\
\frac{d I_{1}^{h}}{d t}=\beta_{1}^{h} S_{1}^{h} I_{1}^{v}-\left(\gamma_{1}^{h}+d_{1}^{h}\right) I_{1}^{h}+m_{12}^{I} I_{2}^{h}-m_{21}^{I} I_{1}^{h} \\
\frac{d I_{1}^{v}}{d t}=\beta_{1}^{v}\left(N_{1}^{v}-I_{1}^{v}\right) I_{1}^{h}-d_{1}^{v} I_{1}^{v}
\end{gathered}
$$

The Jacobian matrix of the system of equations (2.8) at the DFE is obtained as

$$
J_{1}=\left[\begin{array}{ccc}
-d_{1}^{h}-m_{21}^{S} & 0 & \beta_{1}^{h} N_{1}^{h} \\
0 & -d_{1}^{h}-\gamma_{1}^{h}-m_{21}^{I} & \beta_{1}^{h} N_{1}^{h} \\
0 & \beta_{1}^{v} N_{1}^{v} & -d_{1}^{v}
\end{array}\right]
$$

The corresponding Characteristic Polynomial is

$\left(\lambda+d_{1}^{h}+m_{21}^{S}\right)\left[\lambda^{2}+\left(d_{1}^{v}+\gamma_{1}^{h}+d_{1}^{h}+m_{21}^{I}\right) \lambda+d_{1}^{v}\left(m_{21}^{I}+d_{1}^{h}+\gamma_{1}^{h}\right)\left(1-R_{01}^{2}\right)\right]=0$

From characteristic polynomial (2.9), it is observed that first eigen value is $d_{i}^{h}+m_{21}^{S}$ which is negative.

Next,

$$
\lambda^{2}+\left(d_{1}^{v}+\gamma_{1}^{h}+d_{1}^{h}+m_{21}^{I}\right) \lambda+d_{1}^{v}\left(m_{21}^{I}+d_{1}^{h}+\gamma_{1}^{h}\right)\left(1-R_{01}^{2}\right)
$$

The two conditions of Routh Hurwitz Criteria (Brauer and Chavez (2012)), for local asymptotical stability of second order characteristic polynomial $\lambda^{2}+a_{1} \lambda+a_{2}=0$ are $a_{1}>0$ and $a_{2}>0$.

We have, $a_{1}=d_{1}^{v}+\gamma_{1}^{h}+d_{1}^{h}+m_{21}^{I}, a_{2}=$ $d_{1}^{v}\left(m_{21}^{I}+d_{1}^{h}+\gamma_{1}^{h}\right)\left(1-R_{01}^{2}\right)$.Here, $a_{1}$ is always positive. Also, $a_{2}>0$ if $R_{01}<1$. Hence, if $R_{01}<1$, the eigenvalues will have negative real parts, and the disease-free equilibrium point becomes asymptotically stable.

If $R_{01}>1$, then the two eigenvalues of equation (3.0) are one negative and one positive real number. So, the disease-free equilibrium point becomes unstable if $R_{01}>$ 1.

\section{For Patch 2}

Theorem 4: The disease-free equilibrium point is locally asymptotically stable if $R_{02}<$ 1 and unstable if $R_{02}>1$.

\section{Numerical Results and Discussions}

Two patches are assumed to have different disease prevalence. Patch 1 is assumed to have high disease transmission rate, and $R_{01}>1$ and patch 2 is assumed to have low disease transmission rate and $R_{01}<1$. Some numerical simulations are observed with different travel rates of human populations to study the transmission dynamics of dengue disease.

Fig 2 depicts the dynamics of susceptible humans of patch 1 and patch 2 against time. Susceptible host population decreases rapidly with the increase in time in patch 1 . Meanwhile, susceptible host population in patch 2 increases initially and then starts decreasing due to interaction of susceptible hosts with infected mosquitoes and their natural deaths. Since patch 2 is assumed low prevalence patch, the number of susceptible hosts in patches 2 increases initially.

Dynamics of infected host population is observed in Fig 3. As all humans are assumed susceptible to the disease, the number of 
infected hosts increases due to the contact of susceptible hosts with infected mosquitoes. Eventually, the population size of infected

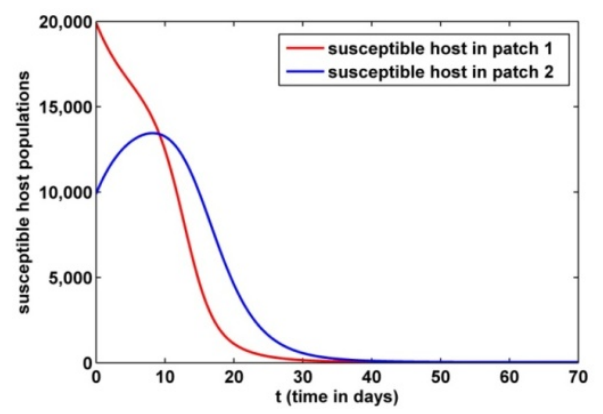

Fig. 2. Dynamics of the susceptible host population.

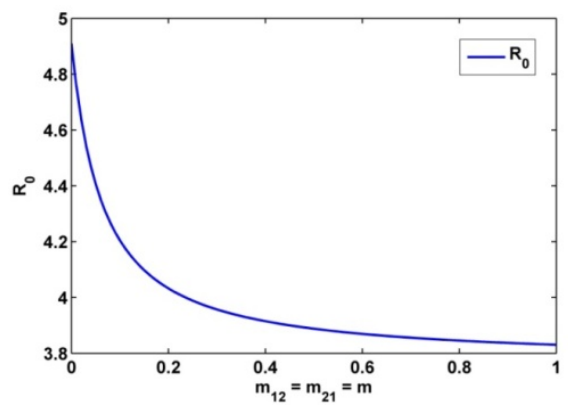

Fig. 4. Basic reproduction number with $m_{12}^{I}=m_{21}^{I}$.

If the travel rates are same (Fig 4), the increase in values of travel rate, $m$ leads to a reduction in the values of the basic reproduction number, $R_{0}$ reducing the dominancy of the disease.

Fig 5 shows the change in values of basic reproduction number, $R_{0}$ with increasing values of $m_{12}$ with fixed values of $m_{21}$. The figure shows that the value of $R_{0}$ decreases hosts in both patches becomes zero due to the recovery of infected humans from the disease and due to their natural death.

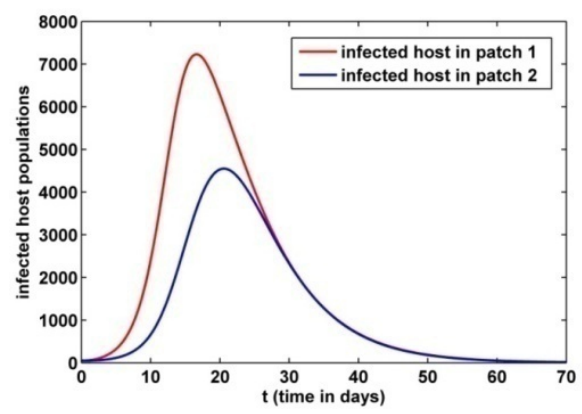

Fig. 3. Dynamics of the infected host population.

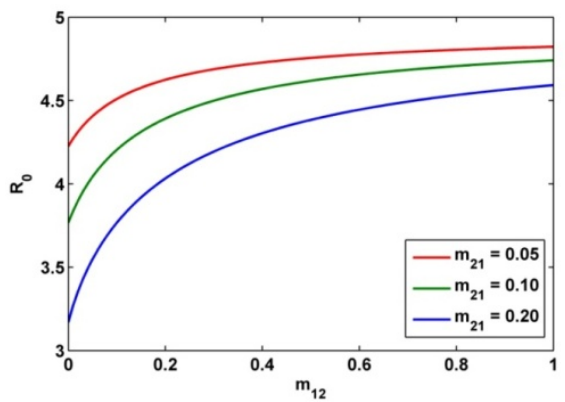

Fig. 5. Basic reproduction number with $m_{12}^{I}$ and fixed values of $m_{21}^{I}$.

with increasing values of $m_{12}$. At the same time, the value of $R_{0}$ decreases with increasing values of $m_{21}$. It shows that the increase in travel rate to low prevalence patch 2 from high prevalence patch 1 reduces the prevalence of the disease. Increase in travel rate to high prevalence patch 1 from low prevalence patch 2 enhances the prevalence of the disease (Fig 6). 


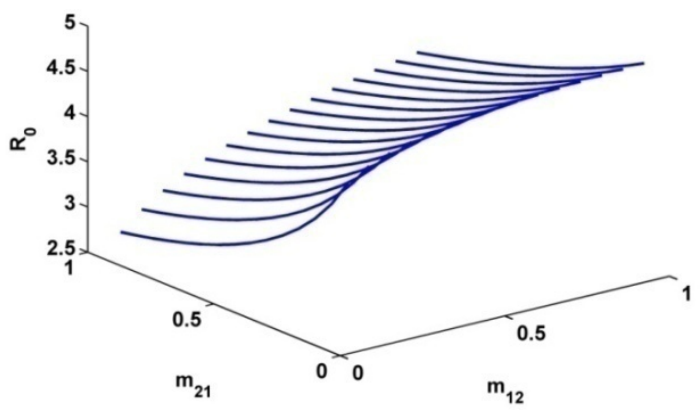

Fig. 6. Basic reproduction number with $m_{12}^{I}$ and $m_{21}^{I}$.

\section{Special Cases}

When infected humans of low prevalence patch 2 are restricted to travel to high prevalence patch 1 (i.e. $m_{12}^{I}=0$ and all other parameters same) and the infected humans of high prevalence patch 1 are allowed to travel to low prevalence patch 2 , it is observed that there are more infected hosts in low prevalence patch 2 than in high prevalence patch 1. (Fig 7). The figure shows that if the infected people of the only high prevalent patch are allowed to travel to low disease prevalence patch, the low disease prevalence patch becomes more disease prevalent.

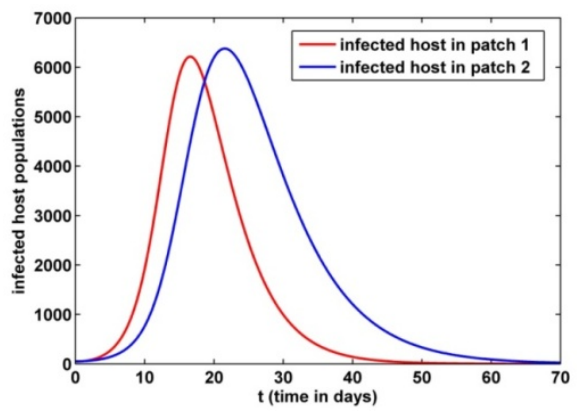

Fig. 7. Dynamics of infected host population with $m_{12}^{I}=0$.

\section{Conclusion}

Travelling of human from one place to another place can change the whole dynamics of dengue disease transmission. Restricting
Fig 8 shows the dynamics of infected host population when the infected people from high disease prevalence patch 1 are restricted to travel to low disease prevalence patch 2 (i.e., $m_{21}^{I}=0$ and all other parameters same) and the infected people from low disease prevalence patch 2 are allowed to travel to high prevalence patch 1 , it is observed that very few people are remained infected in low disease prevalence patch 2 . Thus, restricting the travel of infected humans from high disease prevalence patch to the low disease prevalence patch help in making the low disease prevalence patch even less disease prevalent.

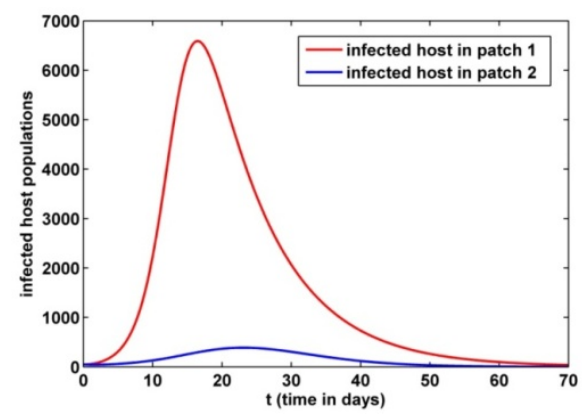

Fig. 8. Dynamics of infected host population with $m_{21}^{I}=0$.

the travel of infected hosts can lead to controlling the disease from being endemic.

Present work discusses the impacts of travelling of human from one patch to the 
other patch. Basic reproduction number is seen higher when there is a higher travel rate from low prevalence patch to the high prevalence patch. The basic reproduction number is seen lowered when there is higher travel rate from high prevalence patch to the low prevalence patch.

Restriction of travel from low prevalence patch to the high prevalence patch when the travel from high prevalence patch to low prevalence patch is allowed, increases the number of infectives in low prevalence patch. Restriction of travel from high prevalence patch to the low prevalence patch when the travel from low prevalence patch to high prevalence patch is allowed, decreases the number of infectives significantly in low prevalence patch. Thus, restricting the travel of infected humans from the high prevalence patch to low prevalence patch could result in low prevalence patch becoming even less disease prevalent.

\section{References}

Arino, J. and P. van den Driessche, 2003. A multicity epidemic model. Math. Popul. Studies. 10: 175 - 193.

Arino, J. and P. Van den Driessche, 2006. Disease spread in meta populations. Field institute communications. 48: 1 - 12 .

Berman, A. and R. J. Plemmons, 1979. Nonnegative matrices in mathematical sciences. Academic Press, New York.

Brauer, F. and C. Castillo Chavez, 2012. Mathematical models in population biology and epidemiology. Springer, New York.

Diekmann, O., J. A. P. Heesterbeek and J. A. J. Metz, 1990. On the definition and computation of the basic reproduction ratio $R_{0}$ in models for infectious diseases in heterogeneous populations. Journal of Mathematical Biology. 28: 365 - 382.

Esteva, L. and C. Vargas, 1998. Analysis of a dengue disease transmission model. Math. Bio-sciences. 150: $131-151$.

Esteva, L. and C. Vargas, 1999. A model for dengue disease with variable human population. Math. Biosciences. 38: 220 - 240.

Gakkhar, S. and N. C. Chavda, 2013. Impact of awareness on the spread of dengue infection in the human population. Applied Mathematics 4: 142 - 147.

Gubler, D., 1998. Dengue and dengue hemorrhagic fever. Clin Microbiol Rev, 3: 480 - 496.
Hsieh, Y. H., P. van den Driessche, and L. Wang, 2007. Impact of travel between patches for the spatial spread of disease. Bulletin of mathematical biology. 69: 1355 - 1375 .

Kermack, W. O. and A. G. McKendrick, 1927. A contribution to the mathematical theory of epidemics. Proceedings of the Royal Society of London. 115: 700 - 721.

Kongnuy, R., E. Naowanich, and P. Pongsumpun, 2011. Analysis of a dengue disease transmission with clinical diagnosis in Thailand. International Journal of Mathematical Models and Methods in Applied Sciences. 5: 594 601.

Maidana, N. A. and H. M. Yang, 2007. A spatial model to describe the dengue propagation. Tend. Mat. Apl. Comput. 8: 83 - 92.

Murray, J. D., 2003. Mathematical biology - II, spatial models and biomedical applications. Springer-Verlag, Berlin.

Phaijoo, G. R. and D. B. Gurung, 2015. Mathematical study of dengue fever with and without awareness in the host population. International Journal of Advanced Engineering Research and Applications (IJAERA). 1: 239 - 245.

Phaijoo, G. R. and D. B. Gurung, 2015. Mathematical study of biting rates of mosquitoes in the transmission of dengue disease, Kathmandu University., Journal of Science, Engineering and Technology (KUSET). 11: 25 - 33.

Pinho, S. T. R., C. P. Ferreira, L. Esteva, F. R. Barreto, V. C. Morato E Silva, and M. G. L. Teixeira, 2010. Modelling the dynamics of dengue real epidemics. Phil. Trans. R. Soci. 368: 5679 - 5693.

Pongsumpun, P., 2008. Mathematical model of dengue disease with the incubation period of the virus. World Academy of Sc. Engg and Tech. 44: 328 - 332.

Soewono, E. and A. K. Supriatna, 2001. A twodimensional model for the transmission of dengue fever disease. Bull. Malaysian Math. Sc. Soc. 24: 49 - 57.

van den Driessche, P. and J. Watmough, 2002. Reproduction numbers and sub-threshold endemic equilibria for compartmental models of disease transmission. Math. Biosci. 180: 29 -48 .

Wang, W. and G. Mulone, 2003. The threshold of disease transmission in a patch environment. J. Math. Anal. Appl. 285: 321 - 335.

Wang, W. and X. Zhao, 2004. An epidemic model in a patchy environment. Math. Biosci. 112: $97-112$.

World Health Organization, 2012. Global strategy for dengue prevention and control 2012 2020. 